\title{
SUPERSYMMETRY AND HOMOGENEITY OF M-THEORY BACKGROUNDS
}

\author{
JOSÉ FIGUEROA-O'FARRILL, PATRICK MEESSEN, AND SIMON PHILIP
}

\begin{abstract}
We describe the construction of a Lie superalgebra associated to an arbitrary supersymmetric M-theory background, and discuss some examples. We prove that for backgrounds with more than 24 supercharges, the bosonic subalgebra acts locally transitively. In particular, we prove that backgrounds with more than 24 supersymmetries are necessarily (locally) homogeneous.
\end{abstract}

\section{Contents}

1. Introduction

2. Supersymmetric M-theory backgrounds

3. Killing vectors

4. The Killing superalgebra

5. Some examples

5.1. Purely gravitational backgrounds

5.2. Branes

6. Supersymmetry and homogeneity

6.1. Homogeneous backgrounds

6.2. Local homogeneity of $24+$ backgrounds

7. Conclusions

Acknowledgments

Appendix A. Clifford algebra conventions

Appendix B. Homogeneity of some $16+$ discrete quotients

B.1. A family of $\nu=\frac{3}{4}$ quotients

B.2. A family of $\nu=\frac{9}{16}$ quotients

Appendix C. A plane wave solution with 22 supercharges

References

\section{INTRODUCTION}

The amount of preserved supersymmetry is an important invariant of a supergravity background; one which has played a pivotal role in the investigations on duality in string theory. This invariant, usually specified as a fraction $\nu$ of the supersymmetry of the theory, admits two complementary refinements: the holonomy representation of the superconnection defined by the variation of the gravitino on the one hand, and the supersymmetry superalgebra on the other. One can recover $\nu$ from either of these two: from the dimension of the invariant subspace in the holonomy representation, or from the dimension of the odd subspace in the superalgebra. The holonomy representation and the supersymmetry superalgebra are not unrelated 1, 2]; although precisely what this relation is remains to be elucidated.

EMPG-04-09, CERN-PH-TH/2004-145. 
Concentrating on the supersymmetry superalgebra for a moment, the seemingly trivial fact that supersymmetries give rise to symmetries suggests that the more supersymmetric a background, the more 'symmetric' it ought to be. Indeed, the maximally supersymmetric backgrounds are all symmetric spaces [3], hence in particular they are homogeneous. Is is therefore natural to ask how much supersymmetry must a background preserve for it to be automatically homogeneous. In other words, is there a critical fraction $\nu_{c}$, such that if a background preserves a fraction $\nu>\nu_{c}$ of the supersymmetry then it is guaranteed to be homogeneous?

Let us concentrate for definiteness on M-theory backgrounds; that is, bosonic solutions of the equations of motion of eleven-dimensional supergravity [4, 5]. Based on known examples and some indirect arguments that we will review presently, a natural conjecture might be that $\nu_{c}=\frac{1}{2}$. Indeed, there are plenty of $\nu=\frac{1}{2}$ backgrounds which are not homogeneous, for example, the elementary $\frac{1}{2}$-BPS backgrounds: the generic M-wave [6], the M-branes [7, 8] and the Kaluza-Klein monopole [9, 10, 11] are not homogeneous; whence $\nu_{c} \geq \frac{1}{2}$. On the other hand, all known examples of backgrounds with $\nu>\frac{1}{2}$, hereafter denoted $16+$, are homogeneous. These examples include the maximally supersymmetric solutions: flat space, the Freund-Rubin backgrounds $\mathrm{AdS}_{4} \times S^{7}$ and $S^{4} \times \mathrm{AdS}_{7}$ [12, and the KowalskiGlikman wave [13, 14]; discrete cyclic quotients [15] of $\mathrm{AdS}_{4} \times S^{7}$ with $\nu=\frac{3}{4}$ and $\nu=\frac{9}{16}$; the Gödel backgrounds [16, 17]; and a number of plane waves, both symmetric [18, 19, 20, 21, 22] and time-dependent [23]. The proof of the homogeneity of the discrete cyclic quotients is presented in Appendix B for the first time, whereas Appendix C] contains a novel non-symmetric plane wave solution with 22 supercharges.

An indirect argument supporting the $\nu_{c}=\frac{1}{2}$ "conjecture" would be that $16+$ plane waves are known to be homogeneous [23]. However as illustrated in [24, homogeneity is not a hereditary property [25] of the plane-wave limit [26, 27]. Therefore one cannot argue that their homogeneity can be explained a posteriori by the hereditary property of supersymmetry 28, just like the plane-wave limit explains [29] the existence of the maximally supersymmetric plane waves [13, 30, 31].

In this paper we will prove that every $24+$ M-theory background is (locally) homogeneous.

This paper is organised as follows. After briefly reviewing the definition of a supersymmetric M-theory background in Section 2 and Kostant's approach to Killing vectors in 3, we define the Killing superalgebra of a supersymmetric M-theory background and prove that it is always a Lie superalgebra. Special cases of this construction have appeared in [32, 33, 34, 35, 36, 14, 30, 37, but in Section 4 we treat the general case. In Section 5 we compute the Killing superalgebra of some standard backgrounds. In Section [6 we define different notions of homogeneity for supergravity backgrounds and prove that $24+$ backgrounds are locally homogeneous. In Section 7 we offer some conclusions. The paper ends with several appendices. Appendix A contains our conventions for the Clifford algebra needed for the calculations in this paper. Appendix $\mathrm{B}$ discusses the homogeneity and the Killing superalgebras of some 16+ discrete quotients, whereas Appendix C presents a new time-dependent $16+$ homogeneous plane wave.

\section{Supersymmetric M-THEORY BACKGROUNDS}

Let $(M, g, F)$ be a classical M-theory background, where $(M, g)$ is a connected eleven-dimensional lorentzian spin manifold and $F$ is a closed four-form, subject to the well-known field equations whose explicit form are of no consequence in what follows. 
Let $\mathcal{S}$ denote the bundle of spinors of this background. (Our Clifford conventions are explained in Appendix $\mathrm{A}$.) The bundle $\mathcal{S}$ is a bundle of Clifford modules, modelled locally on an irreducible $\mathrm{C} \ell(1,10)$-module. There are two such modules up to isomorphism: they are both real and 32-dimensional and are distinguished by the action of the centre of $\mathrm{C} \ell(1,10)$, which is generated by the volume form. Our formulae are valid for the Clifford module on which the action of the centre of $\mathrm{C} \ell(1,10)$ is nontrivial. There is an equivalent version of the theory for the other choice of Clifford module, in which the supersymmetry transformations will differ by some signs.

The variation of the gravitino, after setting the gravitino to zero, defines a connection $\mathcal{D}$ on $\mathcal{S}$, given by

$$
\mathcal{D}_{X}=\nabla_{X}+\frac{1}{6} \iota_{X} F+\frac{1}{12} X^{b} \wedge F
$$

where $X^{b}$ is the one-form dual to $X$ and differential forms act on spinors via the Clifford action as reviewed in Appendix A, The difference in sign from, say, 38, is due to our using a mostly minus metric, which changes the sign of the musical isomorphism $b$.

Nonzero sections of $\mathcal{S}$ which are parallel relative to $\mathcal{D}$ are called Killing spinors, and an M-theory background $(M, g, F)$ admitting Killing spinors is said to be supersymmetric. Since $M$ is connected, a Killing spinor is uniquely determined by its value at a point: its value at any other point is obtained by parallel translating with respect to the connection $\mathcal{D}$. Since Killing spinors are parallel with respect to $\mathcal{D}$, this does not depend on the path. Killing spinors therefore define a real sub-bundle $W \subset \mathcal{S}$, where for $p \in M, W_{p} \subset \mathcal{S}_{p}$ is the subspace spanned by the values of all Killing spinors at $p$. The rank of $W$ is equal to $32 \nu$, where $\nu$ is the fraction of supersymmetry preserved by the background.

\section{Killing veCtors}

Whereas Killing spinors are uniquely determined by their value at a point, to specify a Killing vector one requires its value at a point and that of its first derivative. Indeed, as explained for example in [39, 25, Killing vectors are in one-to-one correspondence with parallel sections of the bundle

$$
\mathcal{E}=T M \oplus \mathfrak{s o}(T M),
$$

where $\mathfrak{s o}(T M)$ is the bundle of skew-symmetric endomorphisms (relative to $g$ ) of the tangent bundle, and where the connection is the one defining the so-called Killing transport [39, 25]. Let us review this now.

Let $(M, g)$ be a connected pseudo-riemannian manifold and $\xi$ a vector field. Let $A_{\xi}: T M \rightarrow T M$ be defined by $A_{\xi} X=-\nabla_{X} \xi$. Then $\xi$ is a Killing vector if and only if $A_{\xi}$ is skew-symmetric relative to the metric, denoted here $\langle-,-\rangle$,

$$
\left\langle A_{\xi} X, Y\right\rangle=-\left\langle A_{\xi} Y, X\right\rangle \text {. }
$$

Killing's identity says that

$$
\nabla_{X} A_{\xi}=R(X, \xi),
$$

where $R(X, Y): T M \rightarrow T M$ is defined by

$$
R(X, Y) Z=\nabla_{[X, Y]} Z-\nabla_{X} \nabla_{Y} Z+\nabla_{Y} \nabla_{X} Z
$$

Proof. Notice that

$$
\begin{aligned}
\left(\nabla_{X} A_{\xi}\right) Y & =\nabla_{X} A_{\xi} Y-A_{\xi} \nabla_{X} Y \\
& =-\nabla_{X} \nabla_{Y} \xi+\nabla_{\nabla_{X} Y} \xi
\end{aligned}
$$


whence

$$
\begin{aligned}
\left(\nabla_{X} A_{\xi}\right) Y-\left(\nabla_{Y} A_{\xi}\right) X & =-\nabla_{X} \nabla_{Y} \xi+\nabla_{\nabla_{X} Y} \xi+\nabla_{Y} \nabla_{X} \xi-\nabla_{\nabla_{Y} X} \xi \\
& =-\nabla_{X} \nabla_{Y} \xi+\nabla_{Y} \nabla_{X} \xi+\nabla_{[X, Y]} \xi \\
& =R(X, Y) \xi \\
& =R(X, \xi) Y-R(Y, \xi) X
\end{aligned}
$$

where we have used the algebraic Bianchi identity

$$
R(X, Y) Z+R(Y, Z) X+R(Z, X) Y=0 .
$$

This means that

$$
\left(\nabla_{X} A_{\xi}\right)(Y)-R(X, \xi) Y
$$

is symmetric in $X \leftrightarrow Y$. On the other hand,

$$
\left\langle\left(\nabla_{X} A_{\xi}\right) Y-R(X, \xi) Y, Z\right\rangle=-\left\langle\left(\nabla_{X} A_{\xi}\right) Z-R(X, \xi) Z, Y\right\rangle,
$$

whence $\left\langle\left(\nabla_{X} A_{\xi}\right) Y-R(X, \xi) Y, Z\right\rangle=0$.

This means that a Killing vector $\xi$ is uniquely characterised by the data

$$
\left(\xi_{p},-\nabla \xi_{p}\right) \in T_{p} M \oplus \mathfrak{s o}\left(T_{p} M\right),
$$

at any point $p \in M$. Indeed, Killing vectors are in one-to-one correspondence with parallel sections of the bundle $\mathcal{E}=T M \oplus \mathfrak{s o}(T M)$ under the connection

$$
D_{X}\left(\begin{array}{c}
\xi \\
A
\end{array}\right)=\left(\begin{array}{c}
\nabla_{X} \xi+A(X) \\
\nabla_{X} A-R(X, \xi)
\end{array}\right) .
$$

Let $\mathfrak{k}$ denote the space of parallel sections of $\mathcal{E}$. The Lie bracket of Killing vectors induces a Lie algebra structure on $\mathfrak{k}$ as follows. Let $(\xi, A)$ and $(\eta, B)$ be parallel sections. Their Lie bracket is given by

$$
[(\xi, A),(\eta, B)]=(A \eta-B \xi,[A, B]+R(\xi, \eta)) .
$$

Proof. By definition,

$$
[(\xi, A),(\eta, B)]=([\xi, \eta],-\nabla[\xi, \eta]) .
$$

Now, the torsionless condition of $\nabla$ means that

$$
[\xi, \eta]=\nabla_{\xi} \eta-\nabla_{\eta} \xi=A \eta-B \xi,
$$

using that $A=-\nabla \xi$ and $B=-\nabla \eta$. Similarly,

$$
\begin{aligned}
-\nabla_{X}[\xi, \eta] & =-\nabla_{X}(A \eta-B \xi) \\
& =-\left(\nabla_{X} A\right) \eta-A \nabla_{X} \eta+\left(\nabla_{X} B\right) \xi+B\left(\nabla_{X} \xi\right) \\
& =-R(X, \xi) \eta+A B X+R(X, \eta) \xi-B A X \\
& =[A, B] X+R(\xi, \eta) X,
\end{aligned}
$$

where we have used Killing's identity (2) and the algebraic Bianchi identity (3).

Now the bundle $\mathcal{E}$ is naturally a bundle of Lie algebras with Lie bracket

$$
[(\xi, A),(\eta, B)]_{\varepsilon}=(A \eta-B \xi,[A, B]) .
$$

Therefore we see that the curvature $R(\xi, \eta)$ measures the failure of this natural Lie bracket to agree with the Lie bracket in $\mathfrak{k}$. Indeed, the bracket on $\mathfrak{k}$ extends to arbitrary sections of $\mathcal{E}$, but it will fail to satisfy the Jacobi identity precisely due to the curvature term.

If $(M, g, F)$ is a supergravity background, then the $F$-preserving elements of $\mathfrak{k}$ define a Lie subalgebra which, anticipating our next topic, will be denoted $\mathfrak{g}_{0}$. 


\section{The Killing superalgebra}

The Killing spinors and the $F$-preserving Killing vectors of a supergravity background $(M, g, F)$ define a Lie superalgebra, which we call the Killing superalgebra of the background.

We shall denote the Killing superalgebra by $\mathfrak{g}=\mathfrak{g}_{0} \oplus \mathfrak{g}_{1}$, where the even subalgebra $\mathfrak{g}_{0}$ is the Lie algebra of $F$-preserving Killing vectors and the odd subspace $\mathfrak{g}_{1}$ consists of (the "oddification" of) the Killing spinors. The grading implies that we must distinguish three types of brackets.

First of all we have the bracket $[-,-]: \mathfrak{g}_{0} \otimes \mathfrak{g}_{0} \rightarrow \mathfrak{g}_{0}$, corresponding to the Lie bracket of Killing vectors defined in (4). It clearly satisfies the Jacobi identity, whence $\mathfrak{g}_{0}$ is a Lie algebra.

The bracket $[-,-]: \mathfrak{g}_{0} \otimes \mathfrak{g}_{1} \rightarrow \mathfrak{g}_{1}$ corresponds to the action of the Killing vectors on the Killing spinors via the spinorial Lie derivative [40. Let $\rho: \mathfrak{s o}(T M) \rightarrow$ End $\mathcal{S}$ denote the spinor representation. Then if $\left(\xi, A_{\xi}\right) \in \mathfrak{k}$, and $\varepsilon \in \mathfrak{g}_{1}$, we define

$$
\left[\left(\xi, A_{\xi}\right), \varepsilon\right]=\nabla_{\xi} \varepsilon+\rho\left(A_{\xi}\right) \varepsilon
$$

where the right-hand side defines the spinorial Lie derivative $\mathcal{L}_{\xi}$. If $\left(\xi, A_{\xi}\right) \in \mathfrak{g}_{0}$, then the right-hand side will again be in $\mathfrak{g}_{1}$ since for all vector fields $X$, one has

$$
\left[\mathcal{L}_{\xi}, \mathcal{D}_{X}\right]=\mathcal{D}_{[\xi, X]} .
$$

The spinorial Lie derivative satisfies

$$
\mathcal{L}_{X} \mathcal{L}_{Y} \varepsilon-\mathcal{L}_{Y} \mathcal{L}_{X} \varepsilon=\mathcal{L}_{[X, Y]} \varepsilon
$$

Proof. Applying (5) and dropping $\rho$ from the notation, we find

$$
\begin{aligned}
{\left[\mathcal{L}_{X}, \mathcal{L}_{Y}\right] \varepsilon } & =\mathcal{L}_{X}\left(\nabla_{Y} \varepsilon+A_{Y} \varepsilon\right)-\mathcal{L}_{Y}\left(\nabla_{X} \varepsilon+A_{X} \varepsilon\right) \\
& =\nabla_{X} \nabla_{Y} \varepsilon+A_{X} \nabla_{Y} \varepsilon+\nabla_{X}\left(A_{Y} \varepsilon\right)+A_{X} A_{Y} \varepsilon-(X \leftrightarrow Y) \\
& =\nabla_{X} \nabla_{Y} \varepsilon-\nabla_{Y} \nabla_{X} \varepsilon+\left[A_{X}, A_{Y}\right] \varepsilon+\left(\nabla_{X} A_{Y}\right) \varepsilon-\left(\nabla_{Y} A_{X}\right) \varepsilon .
\end{aligned}
$$

We now use that

$$
\left[\nabla_{X}, \nabla_{Y}\right] \varepsilon=\nabla_{[X, Y]} \varepsilon-R(X, Y) \varepsilon
$$

and Killing's identity (2) repeatedly to arrive at

$$
\begin{aligned}
{\left[\mathcal{L}_{X}, \mathcal{L}_{Y}\right] \varepsilon } & =\nabla_{[X, Y]} \varepsilon+\left[A_{X}, A_{Y}\right] \varepsilon+R(X, Y) \varepsilon \\
& =\nabla_{[X, Y]} \varepsilon+A_{[X, Y]} \varepsilon \\
& =\mathcal{L}_{[X, Y]} \varepsilon
\end{aligned}
$$

Equation (6) is equivalent to the $\left[\mathfrak{g}_{0}, \mathfrak{g}_{0}, \mathfrak{g}_{1}\right]$-Jacobi identity.

The bracket $[-,-]: \mathfrak{g}_{1} \otimes \mathfrak{g}_{1} \rightarrow \mathfrak{g}_{0}$ is induced from the tensor-square of the corresponding Killing spinors. Indeed, we have a map

$$
\xi: \mathcal{S} \otimes \mathcal{S} \rightarrow T M
$$

which takes two spinors $\varepsilon_{1}$ and $\varepsilon_{2}$ and produces a vector field $\xi\left(\varepsilon_{1}, \varepsilon_{2}\right)$ defined as the unique vector field such that for all other vector fields $Y$,

$$
\left\langle\xi\left(\varepsilon_{1}, \varepsilon_{2}\right), Y\right\rangle=\left(\varepsilon_{1}, Y^{\mathrm{b}} \cdot \varepsilon_{2}\right) .
$$

The map (7) is defined on all spinors, but its restriction to Killing spinors has a crucial property: namely, that if $\varepsilon_{1}$ and $\varepsilon_{2}$ are Killing spinors, then $X=\xi\left(\varepsilon_{1}, \varepsilon_{2}\right)$ is a Killing vector, so that $\mathcal{L}_{X} g=0$, which in addition [41] preserves $F$. 
Proof. Let $\varepsilon_{i}, i=1,2$, be Killing spinors. Then for all vectors $X, Y$, we have

$$
\begin{aligned}
\left\langle\nabla_{X} \xi\left(\varepsilon_{1}, \varepsilon_{2}\right), Y\right\rangle & =X\left\langle\xi\left(\varepsilon_{1}, \varepsilon_{2}\right), Y\right\rangle-\left\langle\xi\left(\varepsilon_{1}, \varepsilon_{2}\right), \nabla_{X} Y\right\rangle \\
& =X\left(\varepsilon_{1}, Y^{b} \cdot \varepsilon_{2}\right)-\left(\varepsilon_{1}, \nabla_{X} Y^{b} \cdot \varepsilon_{2}\right) \\
& =\left(\nabla_{X} \varepsilon_{1}, Y^{b} \cdot \varepsilon_{2}\right)+\left(\varepsilon_{1}, Y^{b} \cdot \nabla_{X} \varepsilon_{2}\right) .
\end{aligned}
$$

Using that $\mathcal{D} \varepsilon_{i}=0$, we can rewrite this as

$$
\left\langle\nabla_{X} \xi\left(\varepsilon_{1}, \varepsilon_{2}\right), Y\right\rangle=\left(\varepsilon_{1}, \Omega_{X}^{*} \cdot Y^{b} \cdot \varepsilon_{2}\right)+\left(\varepsilon_{1}, Y^{b} \cdot \Omega_{X} \cdot \varepsilon_{2}\right)
$$

where

$$
\Omega_{X}=-\frac{1}{12} X^{b} \wedge F-\frac{1}{6} \iota_{X} F
$$

and $\Omega_{X}^{*}=\frac{1}{12} X^{b} \wedge F-\frac{1}{6} \iota_{X} F$ is its symplectic adjoint as defined in (25). Using equations (19) and (20) in Appendix $\mathrm{A}$, we arrive at

$$
\left\langle\nabla_{X} \xi\left(\varepsilon_{1}, \varepsilon_{2}\right), Y\right\rangle=-\frac{1}{3}\left(\varepsilon_{1}, \iota_{X} \iota_{Y} F \cdot \varepsilon_{2}\right)+\frac{1}{6}\left(\varepsilon_{1}, X^{\mathrm{b}} \wedge Y^{\mathrm{b}} \wedge F \cdot \varepsilon_{2}\right),
$$

which is manifestly skew-symmetric in $X$ and $Y$, showing that $\xi\left(\varepsilon_{1}, \varepsilon_{2}\right)$ is a Killing vector.

Now define a 2 -form $B$ by

$$
B(X, Y)=\left(\varepsilon_{1}, X^{b} \wedge Y^{b} \cdot \varepsilon_{2}\right),
$$

and let us compute its covariant derivative. By definition,

$$
\begin{aligned}
\left(\nabla_{Z} B\right)(X, Y) & =\left(\nabla_{Z} \varepsilon_{1}, X^{b} \wedge Y^{b} \cdot \varepsilon_{2}\right)+\left(\varepsilon_{1}, X^{b} \wedge Y^{b} \cdot \nabla_{Z} \varepsilon_{2}\right) \\
& =\left(\Omega_{Z} \varepsilon_{1}, X^{b} \wedge Y^{b} \cdot \varepsilon_{2}\right)+\left(\varepsilon_{1}, X^{b} \wedge Y^{b} \cdot \Omega_{Z} \varepsilon_{2}\right) \\
& =\left(\varepsilon_{1}, \Omega_{Z}^{*} \cdot\left(X^{b} \wedge Y^{b}\right) \cdot \varepsilon_{2}\right)+\left(\varepsilon_{1},\left(X^{b} \wedge Y^{b}\right) \cdot \Omega_{Z} \varepsilon_{2}\right) .
\end{aligned}
$$

Using equations (21) and (22) in Appendix $\mathrm{A}$, we arrive at

$$
\begin{aligned}
\left(\nabla_{Z} B\right)(X, Y) & =\frac{1}{6} g(Y, Z)\left(\varepsilon_{1}, X^{b} \wedge F \cdot \varepsilon_{2}\right)-\frac{1}{6} g(X, Z)\left(\varepsilon_{1}, Y^{b} \wedge F \cdot \varepsilon_{2}\right) \\
+\frac{1}{6}\left(\varepsilon_{1}, Y^{b} \wedge Z^{b}\right. & \left.\wedge \iota_{X} F \cdot \varepsilon_{2}\right)+\frac{1}{6}\left(\varepsilon_{1}, Z^{b} \wedge X^{b} \wedge \iota_{Y} F \cdot \varepsilon_{2}\right) \\
& -\frac{1}{3}\left(\varepsilon_{1}, X^{b} \wedge Y^{b} \wedge \iota_{Z} F \cdot \varepsilon_{2}\right)-\frac{1}{3}\left(\varepsilon_{1}, \iota_{X} \iota_{Y} \iota_{Z} F \cdot \varepsilon_{2}\right) .
\end{aligned}
$$

We now alternate this equation to obtain $d B$ :

$$
\begin{aligned}
d B(X, Y, Z) & =\left(\nabla_{X} B\right)(Y, Z)+\left(\nabla_{Y} B\right)(Z, X)+\left(\nabla_{Z} B\right)(X, Y) \\
& =-\left(\varepsilon_{1}, \iota_{X} \iota_{Y} \iota_{Z} F \cdot \varepsilon_{2}\right) .
\end{aligned}
$$

Noticing that

$$
\left(\varepsilon_{1}, \iota_{X} \iota_{Y} \iota_{Z} F \cdot \varepsilon_{2}\right)=F\left(\xi\left(\varepsilon_{1}, \varepsilon_{2}\right), X, Y, Z\right)
$$

we have that

$$
\iota_{\xi\left(\varepsilon_{1}, \varepsilon_{2}\right)} F=-d B .
$$

Since $F$ is closed, this implies that the vector field $\xi\left(\varepsilon_{1}, \varepsilon_{2}\right)$ leaves $F$ invariant.

It is convenient to extend the map $\xi$ to a map

$$
\varphi: \mathcal{S} \otimes \mathcal{S} \rightarrow \mathcal{E}
$$

This maps restricts to a map sending parallel sections (with respect to $\mathcal{D}$ ) of $\mathcal{S} \otimes \mathcal{S}$ to parallel sections (with respect to $D$ ) of $\mathcal{E}$, which we will also denote $\varphi$. The explicit form of this map is given by

$$
\varphi\left(\varepsilon_{1}, \varepsilon_{2}\right)=\left(\xi\left(\varepsilon_{1}, \varepsilon_{2}\right),-\nabla \xi\left(\varepsilon_{1}, \varepsilon_{2}\right)\right),
$$

where $\xi\left(\varepsilon_{1}, \varepsilon_{2}\right)$ and $\nabla \xi\left(\varepsilon_{1}, \varepsilon_{2}\right)$ are given by equations (8) and (9), respectively. The fundamental property of the map $\varphi$ is its equivariance under the action of $\mathfrak{g}_{0}$. In other words,

$$
\left[\left(X, A_{X}\right), \varphi\left(\varepsilon_{1}, \varepsilon_{2}\right)\right]=\varphi\left(\mathcal{L}_{X} \varepsilon_{1}, \varepsilon_{2}\right)+\varphi\left(\varepsilon_{1}, \mathcal{L}_{X} \varepsilon_{2}\right)
$$


Equivalently, for all vector fields $Y$ (not necessarily Killing),

$$
\left\langle\mathcal{L}_{X} \xi\left(\varepsilon_{1}, \varepsilon_{2}\right), Y\right\rangle=\left(\mathcal{L}_{X} \varepsilon_{1}, Y^{b} \cdot \varepsilon_{2}\right)+\left(\varepsilon_{1}, Y^{b} \cdot \mathcal{L}_{X} \varepsilon_{2}\right) .
$$

Proof. Computing the left-hand side, we find

$$
\begin{aligned}
\left\langle\mathcal{L}_{X} \xi\left(\varepsilon_{1}, \varepsilon_{2}\right), Y\right\rangle & =\left\langle\nabla_{X} \xi\left(\varepsilon_{1}, \varepsilon_{2}\right)-\nabla_{\xi\left(\varepsilon_{1}, \varepsilon_{2}\right)} X, Y\right\rangle \\
& =\left(\nabla_{X} \varepsilon_{1}, Y^{b} \cdot \varepsilon_{2}\right)+\left(\varepsilon_{1}, Y^{b} \cdot \nabla_{X} \varepsilon_{2}\right)+\left(\varepsilon_{1}, \nabla_{Y} X^{b} \cdot \varepsilon_{2}\right) .
\end{aligned}
$$

Computing the right-hand side, we obtain

$$
\begin{aligned}
\left(\mathcal{L}_{X} \varepsilon_{1}, Y^{b} \cdot \varepsilon_{2}\right)+\left(\varepsilon_{1}, Y^{b} \cdot \mathcal{L}_{X} \varepsilon_{2}\right)= & \left(\nabla_{X} \varepsilon_{1}, Y^{b} \cdot \varepsilon_{2}\right)+\left(A_{X} \varepsilon_{1}, Y^{b} \cdot \varepsilon_{2}\right) \\
& +\left(\varepsilon_{1}, Y^{b} \cdot \nabla_{X} \varepsilon_{2}\right)+\left(\varepsilon_{1}, Y^{b} \cdot A_{X} \cdot \varepsilon_{2}\right) .
\end{aligned}
$$

The difference is therefore

$$
\left(\varepsilon_{1}, \nabla_{Y} X^{b} \cdot \varepsilon_{2}\right)+\left(\varepsilon_{1}, A_{X} \cdot Y^{b} \cdot \varepsilon_{2}\right)-\left(\varepsilon_{1}, Y^{b} \cdot A_{X} \cdot \varepsilon_{2}\right),
$$

which is easily seen to vanish as a consequence of the identity

$$
\left[A_{X}, Y\right]=A_{X}(Y)=-\nabla_{Y} X .
$$

Equation (12) is precisely the $\left[\mathfrak{g}_{0}, \mathfrak{g}_{1}, \mathfrak{g}_{1}\right]$-Jacobi identity. It also implies that $\left[\mathfrak{g}_{1}, \mathfrak{g}_{1}\right] \subset \mathfrak{g}_{0}$ is an ideal, which is a general fact of superalgebras. In other words, $\mathfrak{g}_{1}$ generates an ideal $\left[\mathfrak{g}_{1}, \mathfrak{g}_{1}\right] \oplus \mathfrak{g}_{1} \subset \mathfrak{g}$.

Finally we consider the $\left[\mathfrak{g}_{1}, \mathfrak{g}_{1}, \mathfrak{g}_{1}\right]$-Jacobi identity. This is equivalent to the vanishing of a $\mathfrak{g}_{0}$-equivariant symmetric trilinear map $J: S^{3} \mathfrak{g}_{1} \rightarrow \mathfrak{g}_{1}$, defined by

$$
J\left(\varepsilon_{1}, \varepsilon_{2}, \varepsilon_{3}\right):=\mathcal{L}_{\xi\left(\varepsilon_{1}, \varepsilon_{2}\right)} \varepsilon_{3}+\mathcal{L}_{\xi\left(\varepsilon_{2}, \varepsilon_{3}\right)} \varepsilon_{1}+\mathcal{L}_{\xi\left(\varepsilon_{3}, \varepsilon_{1}\right)} \varepsilon_{2}
$$

The vanishing of $J$ is equivalent to

$$
\mathcal{L}_{\xi(\varepsilon, \varepsilon)} \varepsilon=0
$$

for all Killing spinors $\varepsilon$.

Proof. Equation (14) is simply $J(\varepsilon, \varepsilon, \varepsilon)=0$, up to an overall factor of 3 . Hence this vanishes when $J$ vanishes. Conversely we can use the standard polarisation tricks; that is, apply (14) to $\varepsilon=\varepsilon_{1}+\varepsilon_{2}+\varepsilon_{3}$ to obtain that $2 J\left(\varepsilon_{1}, \varepsilon_{2}, \varepsilon_{3}\right)=0$.

In other words, the Jacobi identity is equivalent to every Killing spinor being left invariant by the Killing vector obtained by squaring it.

Equation (14) does not involve any derivatives. Indeed, it is equivalent to

$$
\left(2 \iota_{\xi} F+\xi^{b} \wedge F+B \wedge \star F+C \wedge F\right) \cdot \varepsilon=0,
$$

where $\xi^{b}, B$ and $C$ are the 1-, 2- and 5-forms constructed out of the Killing spinor $\varepsilon$, respectively:

$$
\begin{gathered}
\xi^{b}(X)=\left(\varepsilon, X^{b} \cdot \varepsilon\right) \\
B(X, Y)=\left(\varepsilon, X^{b} \wedge Y^{b} \cdot \varepsilon\right) \\
C\left(X_{1}, \ldots, X_{5}\right)=\left(\varepsilon, X_{1}^{b} \wedge \cdots \wedge X_{5}^{b} \cdot \varepsilon\right) .
\end{gathered}
$$

Equation (14) is clearly linear in $F$ and cubic in $\varepsilon$ and furthermore it is equivariant under the action of $\operatorname{Spin}(1,10)$. As a consequence, it need only be checked for one $(F, \varepsilon)$ in each of the (projectivised) Spin $(1,10)$-orbits of the relevant representation space. Rather than working out the orbit decomposition of this rather large space, we can instead try to prove that this identity holds for all $F$ and for one spinor $\varepsilon$ in each of the (projectivised) Spin $(1,10)$ orbits in the spinor representation. There are two such orbits, distinguished by the causal character of the Killing vector 
associated with $\varepsilon$. This can be checked by computer using an explicit real realisation of $\mathrm{C} \ell(1,10)$. For this it is convenient to unpack (15) further and rewrite it as

$$
\begin{aligned}
F_{a b c d}\left(\frac{2}{3}\left(\varepsilon, \Gamma^{a} \varepsilon\right) \Gamma^{b c d}+\frac{1}{12}(\varepsilon,\right. & \left.\Gamma_{e} \varepsilon\right) \Gamma^{a b c d e} \\
& \left.+\left(\varepsilon, \Gamma^{a b} \varepsilon\right) \Gamma^{c d}+\frac{1}{24}\left(\varepsilon, \Gamma^{a b c d m n} \varepsilon\right) \Gamma_{m n}\right) \varepsilon=0
\end{aligned}
$$

where we have used the Einstein summation convention. Equation (16) has been shown to hold for all $F$ and all $\varepsilon$ using two independent computer calculations: one in Maple and one in Mathematica. The relevant code is available upon request from the authors.

\section{Some EXAmples}

In this section we will discuss several examples of Killing superalgebras for some M-theory backgrounds.

5.1. Purely gravitational backgrounds. We start with those backgrounds where $F=0$. In this case the Killing spinors are parallel relative to the Levi-Cività connection. This means that so are the vectors in $\left[\mathfrak{g}_{1}, \mathfrak{g}_{1}\right]$. In particular, their action on $\mathfrak{g}_{1}$ is trivial. This means that $\left[\mathfrak{g}_{1}, \mathfrak{g}_{1}\right]$ is abelian and, for the purely gravitational backgrounds, they consist of translations.

Examples of such backgrounds are flat space, the M-wave [6], the Kaluza-Klein monopole [9, 10, 11] as well as their generalisations [42]. For flat space, $\left[\mathfrak{g}_{1}, \mathfrak{g}_{1}\right]$ coincides with the translation ideal. For the M-wave, we obtain a one-dimensional ideal spanned by the parallel null vector $v$ in the pp-wave. Indeed, let $u$ be a complementary null vector such that $u \cdot v+v \cdot u=\mathbb{1}$ in the Clifford algebra. Such a vector always exists locally. The Killing spinors $\varepsilon$ satisfy the condition $v \cdot \varepsilon=0$, which means that $\varepsilon=v \cdot u \cdot \varepsilon$. Now let $\varepsilon_{1}, \varepsilon_{2}$ be Killing vectors. If $X \perp v$ then

$$
\begin{aligned}
\left\langle\xi\left(\varepsilon_{1}, \varepsilon_{2}\right), X\right\rangle & =\left(\varepsilon_{1}, X \cdot \varepsilon_{2}\right) \\
& =\left(\varepsilon_{1}, X \cdot v \cdot u \cdot \varepsilon_{2}\right) \\
& =-\left(\varepsilon_{1}, v \cdot X \cdot u \cdot \varepsilon_{2}\right) \\
& =\left(v \cdot \varepsilon_{1}, X \cdot u \cdot \varepsilon_{2}\right) \\
& =0 .
\end{aligned}
$$

Therefore $\xi\left(\varepsilon_{1}, \varepsilon_{2}\right)$ is perpendicular to every vector $X$ which is perpendicular to $v$, whence it is collinear with $v$. Since $v$ and $\xi\left(\varepsilon_{1}, \varepsilon_{2}\right)$ are both parallel, we see that $\xi\left(\varepsilon_{1}, \varepsilon_{2}\right)=c v$ for some constant $c$.

For the Kaluza-Klein monopole and its generalisations, we obtain the translations in the flat factor. Indeed, the geometry here is $\mathbb{R}^{1,10-n} \times X^{n}$ where $X$ is a riemannian manifold admitting parallel spinors and having no flat directions; that is, no parallel vector fields. The possible holonomy groups of $X$ are tabulated in [43] and are given by $\mathrm{SU}(5)$ for $n=10$, any of $\mathrm{Sp}(1) \times \mathrm{Sp}(1) \subset \mathrm{Sp}(2) \subset \mathrm{SU}(4) \subset \mathrm{Spin}(7)$ for $n=8, G_{2}$ for $n=7, \mathrm{SU}(3)$ for $n=6$ and $\mathrm{Sp}(1)=\mathrm{SU}(2)$ for $n=4$. In all cases we obtain that $\left[\mathfrak{g}_{1}, \mathfrak{g}_{1}\right]$ is the translation ideal $\mathbb{R}^{1,10-n}$.

5.2. Branes. For the elementary half-BPS M2- and M5-brane backgrounds one also finds that $\left[\mathfrak{g}_{1}, \mathfrak{g}_{1}\right]$ is the translation ideal $\mathbb{R}^{1, p}$ on the brane. Both backgrounds are geometrically a warped product

$$
g=H^{\alpha} \eta+H^{\beta} \delta,
$$

where $\eta$ is the Minkowski metric on $\mathbb{R}^{1, p}, p=2,5 ; \delta$ is the Euclidean metric on $\mathbb{R}^{q}$, $q=8,5$, respectively; and $H$ is a harmonic function on $\mathbb{R}^{q}$ such that the metric is 
asymptotically flat. The coefficients $\alpha$ and $\beta$ are given in terms of $p$, but we do not need their explicit form. The Killing spinors are given by

$$
\varepsilon=H^{\alpha / 4} \varepsilon_{\infty},
$$

where $\varepsilon_{\infty}$ is a parallel spinor in the asymptotically flat geometry which obeys the algebraic condition

$$
\nu_{\eta} \cdot \varepsilon_{\infty}=\varepsilon_{\infty},
$$

where $\nu_{\eta}$ is the volume form of the Minkowski metric $\eta$. Notice that the same identity is satisfied by $\varepsilon$ itself.

Consider the case of the M2-brane. Here $\nu_{\eta}$ is a 3 -form and hence it is self-adjoint relative to the symplectic structure on the spinor bundle. Let $X$ be perpendicular to the brane world-volume. Then $X \cdot \nu_{\eta}=-\nu_{\eta} \cdot X$, and hence if $\varepsilon_{1}$ and $\varepsilon_{2}$ are Killing spinors,

$$
\begin{aligned}
\left(\varepsilon_{1}, X \cdot \varepsilon_{2}\right) & =\left(\varepsilon_{1}, X \cdot \nu_{\eta} \cdot \varepsilon_{2}\right) \\
& =\left(\varepsilon_{1},-\nu_{\eta} \cdot X \cdot \varepsilon_{2}\right) \\
& =-\left(\nu_{\eta} \cdot \varepsilon_{1}, X \cdot \varepsilon_{2}\right) \\
& =-\left(\varepsilon_{1}, X \cdot \varepsilon_{2}\right) .
\end{aligned}
$$

Therefore $\xi\left(\varepsilon_{1}, \varepsilon_{2}\right)$ is in the double perpendicular of the tangent space to the worldvolume of the brane, whence tangent to the world-volume of the brane. This result is intuitively obvious because this argument works for any harmonic function $H$, even if this function has no symmetries.

A similar calculation shows the analogous result for the M5-brane. Here $\nu_{\eta}$ is a 6 -form, whence it is symplectically skew-adjoint. However, if $X$ is perpendicular to the brane world-volume, now $X \cdot \nu_{\eta}=\nu_{\eta} \cdot X$. A calculation virtually identical to the one above yields that $\xi\left(\varepsilon_{1}, \varepsilon_{2}\right)$ is tangent to the brane world-volume.

Now if $X$ is tangent to the brane world-volume and $\varepsilon$ is a Killing spinor, a quick calculation shows that

$$
\nabla_{X} \varepsilon=\frac{1}{4} \alpha d \log H \cdot X \cdot \varepsilon .
$$

Let $Y=Y_{\|}+Y_{\perp}$ be any vector field, where we have decomposed into parallel and perpendicular components with respect to the brane world-volume, and let $\varepsilon_{1}, \varepsilon_{2}$ be Killing spinors. Then,

$$
\begin{aligned}
\left\langle\nabla_{X} \xi\left(\varepsilon_{1}, \varepsilon_{2}\right), Y\right\rangle & =\left\langle\nabla_{X} \xi\left(\varepsilon_{1}, \varepsilon_{2}\right), Y_{\|}\right\rangle \\
& =\left\langle\xi\left(\nabla_{X} \varepsilon_{1}, \varepsilon_{2}\right), Y_{\|}\right\rangle+\left\langle\xi\left(\varepsilon_{1}, \nabla_{X} \varepsilon_{2}\right), Y_{\|}\right\rangle \\
& =\left(\nabla_{X} \varepsilon_{1}, Y_{\|} \cdot \varepsilon_{2}\right)+\left(\varepsilon_{1}, Y_{\|} \cdot \nabla_{X} \varepsilon_{2}\right) \\
& =\frac{1}{4} \alpha\left(d \log H \cdot X \cdot \varepsilon_{1}, Y_{\|} \cdot \varepsilon_{2}\right)+\frac{1}{4} \alpha\left(\varepsilon_{1}, Y_{\|} \cdot d \log H \cdot X \cdot \varepsilon_{2}\right) \\
& =\frac{1}{4} \alpha\left(d \log H \cdot \varepsilon_{1}, X \cdot Y_{\|} \cdot \varepsilon_{2}\right)+\frac{1}{4} \alpha\left(d \log H \cdot \varepsilon_{1}, Y_{\|} \cdot X \cdot \varepsilon_{2}\right) \\
& =\frac{1}{2} \alpha\langle X, Y\rangle\left(\varepsilon_{1}, d \log H \cdot \varepsilon_{2}\right) \\
& =\frac{1}{2} \alpha\langle X, Y\rangle\left\langle\xi\left(\varepsilon_{1}, \varepsilon_{2}\right), d \log H\right\rangle \\
& =0,
\end{aligned}
$$

where we have used repeatedly that $d \log H$ is perpendicular to the brane world volume. In other words, the Lorentz component of $\xi\left(\varepsilon_{1}, \varepsilon_{2}\right)$ vanishes, whence it is a translation.

Virtually the same argument applies for the M2-brane at a conical singularity 32, where the transverse euclidean metric $\delta$ is replaced by a cone of holonomy contained in $\operatorname{Spin}(7)$. 
A similar argument also works if we curve the world-volume as in [44] and 45. In the case of static brane world-volumes [44, $\left[\mathfrak{g}_{1}, \mathfrak{g}_{1}\right]$ contains the timelike parallel vector, whereas in the case of the indecomposable supersymmetric waves [45, $\left[\mathfrak{g}_{1}, \mathfrak{g}_{1}\right]$ once again coincides with the null parallel vector.

The Killing superalgebras of the Freund-Rubin backgrounds which appear as near-horizon geometries of elementary branes and of branes at conical singularities have been described in 32 .

\section{SUPERSYMMETRY AND HOMOGENEITY}

In this section we will prove that $24+$ backgrounds are locally homogeneous.

6.1. Homogeneous backgrounds. We recall that a background $(M, g, F)$ is homogeneous if the group $G$ of $F$-preserving isometries acts transitively on $M$, so that for any two points $p, q \in M$, there is a $g \in G$ such that $q=g \cdot p$. In supergravity we usually work with local metrics and do not necessarily impose completeness of the background. In this context, the relevant concept is not homogeneity but local transitivity, namely that every $p \in M$ is contained in a neighbourhood $U$ such that for every $q \in U$ there is a local $F$-preserving isometry $g$ such that $q=g \cdot p$. This is equivalent to the existence of a frame consisting of $F$-preserving Killing vectors at every point $p \in M$. This implies that the background is locally homogeneous; that is, that given any $p, q \in M$ there are neighbourhoods $U$ of $p$ and $V$ of $q$ and a local $F$-preserving isometry $g: U \rightarrow V$ such that $g \cdot p=q$.

Proof. Since $M$ is connected, let $\gamma: I=[0,1] \rightarrow M$ be a continuous curve connecting $p$ and $q$. For every $t \in I$, there is a neighbourhood $U_{t} \subset M$ of $\gamma(t)$ with the property that every $r \in U_{t}$ is related to $\gamma(t)$ by a local $F$-preserving isometry. The intersections $U_{t} \cap \gamma(I)$ define an open cover (relative to the subspace topology) for $\gamma(I)$. Since $\gamma$ is continuous, the preimages $V_{t}=\gamma^{-1}\left(U_{t} \cap \gamma(I)\right)$ are an open cover of the interval. Since the interval is compact, there is a finite subcover $V_{i}=V_{t_{i}}$ for $i=0, \ldots, N$ for some $N$. We can further choose that $0=t_{0}<t_{1}<\cdots<t_{N}=1$ and that the successive intersections $V_{i} \cap V_{i+1}$ are nonempty. Then choose $r_{i} \in \gamma\left(V_{i-1}\right) \cap \gamma\left(V_{i}\right)$. By hypothesis, there exist local isometries $g_{i}, h_{i}$ such that $g_{i} \gamma\left(t_{i}\right)=r_{i}$ and $h_{i} \gamma\left(t_{i}\right)=r_{i+1}$. The desired local isometry between $p$ and $q$ is given by

$$
\psi:=g_{N}^{-1} \circ h_{N-1} \circ \cdots \circ g_{2}^{-1} \circ h_{1} \circ g_{1}^{-1} \circ h_{0} .
$$

Finally let $V$ be a small enough open neighbourhood of $q$, so that $U=\psi^{-1}(V)$ is defined. The open set $U$ is a neighbourhood of $p$ and clearly $\psi: U \rightarrow V$.

In the next section we will prove that any background admitting more than 24 supersymmetries, so that $\nu>\frac{3}{4}$, is locally homogeneous. We will prove this only using basic linear algebra, by studying in detail the restriction of the map $\varphi_{p}$ defined in (11) to the subspace $W_{p}$ spanned by the values of the Killing spinors at an arbitrary point $p \in M$ and showing that if $\operatorname{dim} W_{p}>24$ then the component $\xi_{p}$ of this map on $T_{p} M$ is surjective. We remark that this result is stronger than (local) homogeneity in that we are proving that the symmetries which follow from the supersymmetry already act locally transitively. For example, there are homogeneous plane waves admitting only 16 supersymmetries for which the only Killing vector which can be constructed from Killing spinors is the parallel null vector; although the Killing vectors for the homogeneous plane waves with more than 16 supersymmetries can be constructed from the Killing spinors [23. In fact, one can see that for all $16+$ solutions mentioned in the introduction local transitivity is already implied by supersymmetry. 
6.2. Local homogeneity of $\mathbf{2 4}+$ backgrounds. We will fix a point $p \in M$ once and for all. The tangent space $T_{p} M$ with the restriction of the metric $g(p)$ becomes a lorentzian inner product space. We will denote it $V$ and will let $\langle-,-\rangle$ denote the lorentzian inner product and $|-|^{2}$ denote the associated (indefinite) norm. The fibre $\mathcal{S}_{p}$ of the spinor bundle is isomorphic to the irreducible $\mathrm{C} \ell(V)$-module $S$, which is a 32-dimensional real symplectic vector space, with symplectic structure denoted by $(-,-)$ as above. Let $W \subset S$ be the subspace corresponding to the Killing spinors. The map (7) defines a symmetric bilinear map

$$
\xi: S \otimes S \rightarrow V .
$$

We want to show that if $\operatorname{dim} W$ is large enough, then the restriction

$$
\left.\xi\right|_{W}: W \otimes W \rightarrow V
$$

of $\xi$ to $W$ is surjective. This means that $T_{p} M$ is spanned by the values of $F$ preserving Killing vectors. Since $p$ is arbitrary, this will be the case at every point and the background will be locally homogeneous.

Clearly if $W=S$ then $\xi$ is surjective: this follows from the representation theory of the spin group. On the other hand there are examples with $\operatorname{dim} W=16$ which are not homogeneous, hence there has to be a minimal $16<N \leq 32$ such that whenever $\operatorname{dim} W \geq N$, the map $\left.\xi\right|_{W}$ is surjective. We will show that $N=25$.

In our proof we will exploit the fact that $S$ is a symplectic vector space, so it might be convenient to introduce some relevant notation from symplectic linear algebra. Let $W \subset S$ be any vector subspace. The vectors which are symplectically perpendicular to all the vectors in $W$ define a subspace

$$
W^{\perp}=\{\varepsilon \in S \mid(\varepsilon, w)=0 \text { for all } w \in W\} .
$$

Analogous to the case of a euclidean structure, we also have that

$$
\operatorname{dim} W+\operatorname{dim} W^{\perp}=\operatorname{dim} S,
$$

even though $W$ and $W^{\perp}$ are not generally disjoint. For example, every onedimensional subspace is contained in its symplectic perpendicular. The relationship between $W$ and $W^{\perp}$ defines certain types of subspaces. For example, a subspace such that $W \subset W^{\perp}$ is called isotropic. Clearly the dimension of an isotropic subspace is at most half the dimension of $S$. When the dimension is precisely half, so that $W=W^{\perp}, W$ is called lagrangian. At the other extreme, if $W$ and $W^{\perp}$ are disjoint, then $W$ is said to be a symplectic subspace.

As a side remark, we mention the intriguing fact that the Killing spinors for the elementary half-BPS backgrounds define special subspaces: lagrangian in the case of the M5-brane and the M-wave and symplectic in the case of the M2-brane and the Kaluza-Klein monopole. This is somewhat puzzling because the connection $\mathcal{D}$ does not preserve the symplectic structure in general. It does for the purely gravitational backgrounds, whose holonomy group is contained in $\operatorname{Spin}(1,10)$ and hence in $\operatorname{Sp}(32, \mathbb{R})$, but the calculations in [46] show that the holonomy algebras of the M2-brane and M5-brane are not contained in the symplectic subalgebra $\mathfrak{s p}(32, \mathbb{R}) 1$

Let us now proceed with the proof. Let $\operatorname{dim} W>16$, since the known examples already negate anything else. The map $\left.\xi\right|_{W}$ is surjective if and only if the subspace perpendicular to its image is trivial. Equivalently, if and only if the only vector $v \in V$ obeying

$$
\left(\varepsilon_{1}, v \cdot \varepsilon_{2}\right)=0 \quad \text { for all } \varepsilon_{i} \in W
$$

\footnotetext{
${ }^{1}$ We are grateful to George Moutsopoulos for checking this.
} 
is the zero vector $v=0$. Throughout this section we will allow vectors (and not just forms) to act on spinors. By definition, the action of a vector $v$ is simply the Clifford action of the dual one-form $v^{b}$.

Our first observation is that any $v \in V$ satisfying (18) is necessarily null. Indeed, notice that (18) can be rephrased as saying that as a Clifford endomorphism

$$
v: W \rightarrow W^{\perp} .
$$

Since $\operatorname{dim} W>\frac{1}{2} \operatorname{dim} S$, it follows from (17) that $\operatorname{dim} W>\operatorname{dim} W^{\perp}$, whence $v$ must have kernel, purely on dimensional grounds. On the other hand, the Clifford algebra says that $v^{2}=-|v|^{2} \mathbb{1}$, whence $v$ has kernel if and only if $|v|^{2}=0$.

Since in a lorentzian vector space all null subspaces are one-dimensional, we deduce that the subspace perpendicular (relative to $\langle-,-\rangle$ ) to the image of $\xi$ is at most one-dimensional. Moreover, if one-dimensional, it is spanned by a null vector $v \in V$

Our next step is to show that in this case the Clifford endomorphism $v$ has rank 16. From $v^{2}=0$, we see that $\operatorname{im} v \subset \operatorname{ker} v$. To show the reverse inclusion, let $u \in V$ be a complementary null vector such that

$$
u \cdot v+v \cdot u=\mathbb{1} .
$$

(In other words, we can think of $v$ as $\Gamma_{+}$and $u$ as $\Gamma_{-}$. .) Then applying both sides of this identity to a vector $\varepsilon$ annihilated by $v$, we find

$$
\varepsilon=v \cdot u \cdot \varepsilon \in \operatorname{im} v,
$$

whence $\operatorname{ker} v=\operatorname{im} v$. A similar $\operatorname{argument}$ shows that $\operatorname{ker} u=\operatorname{im} u$. Moreover, from (24) it follows that ker $u$ and ker $v$ are complementary lagrangian subspaces of $S$. In particular, $\operatorname{rank} v=\operatorname{dim} \operatorname{im} v=16$.

Now let $U$ be a complementary subspace to $W$, so that $S=W \oplus U$. Relative to this split, the symmetric bilinear form $\beta$, defined by

$$
\beta\left(\varepsilon_{1}, \varepsilon_{2}\right)=\left(\varepsilon_{1}, v \cdot \varepsilon_{2}\right),
$$

has the following matrix

$$
\left(\begin{array}{cc}
0 & A \\
A^{t} & B
\end{array}\right)
$$

where $A: U \rightarrow W, A^{t}: W \rightarrow U$ and $B: U \rightarrow U$ are linear maps. We know that this matrix has rank 16 , since $(-,-)$ is nondegenerate and $v$ has rank 16 . What we will do now is estimate the maximal possible rank in terms of the dimension of $W$.

The kernel of $\beta$ consists of $(w, u) \in W \oplus U$ such that $A u=0$ and $A^{t} w+B u=0$. Notice that $\operatorname{dim} U<\operatorname{dim} W$, whence $\operatorname{rank} A \leq \operatorname{dim} U$. In the case of maximal rank, the only solution of $A u=0$ is $u=0$. In this case the kernel of $\beta$ consists of $(w, 0)$ with $w \in \operatorname{ker} A^{t}$. In other words, the dimensions of the kernels of $\beta$ and of $A^{t}$ agree. Since $A^{t}$ and $A$ have the same rank, $A^{t}$ is onto, whence its kernel has dimension $\operatorname{dim} W-\operatorname{dim} U$. Therefore the rank of $\beta$ is at most $32-\operatorname{dim} W+\operatorname{dim} U=2 \operatorname{dim} U$; but we know that the rank of $\beta$ is 16 , whence $16 \leq 2 \operatorname{dim} U$ or $\operatorname{dim} U \geq 8$. This means that if $\operatorname{dim} U<8$ (equivalently, if $\operatorname{dim} W>24$ ) no such $v$ can exist and the $\left.\operatorname{map} \xi\right|_{W}$ is surjective.

\section{Conclusions}

In this paper we have investigated the relation between symmetry and supersymmetry in supergravity backgrounds, concentrating for definiteness in elevendimensional supergravity. We have shown that the Killing spinors in any such background generate a Lie superalgebra. Strictly speaking they generate an ideal 
of what we call the Killing superalgebra of the background, which may contain additional "accidental" bosonic symmetries. The Killing superalgebra has appeared before in many special cases, but until now there was no general proof that this construction resulted in a Lie superalgebra.

Since supersymmetries generate symmetries, we posed the general question of whether there is a mininum amount of supersymmetry that a solution must preserve for it to be automatically (locally) homogeneous. Homogeneous backgrounds are particularly tractable and a positive answer to that question implies that a classification of homogeneous backgrounds, for example, would automatically imply a classification of solutions preserving more than a certain critical fraction $\nu_{c}$ of supersymmetry.

We have reviewed what is known about backgrounds admitting more than 16 supersymmetries and have observed that all known such backgrounds are homogeneous. Moreover, it is the ideal of the Killing superalgebra generated by the supersymmetries which already acts locally transitively. We have checked this for the known $16+$ solutions and also for some recently discovered ones, included in the appendices.

Finally we have proven that if a solution preserves more than 24 supersymmetries then the ideal of the Killing superalgebra generated by these supersymmetries acts locally transitively on the background. In particular, these $24+$ backgrounds are locally homogeneous.

\section{ACKNOWLEDGMENTS}

The research of JMF is partially funded by the EPSRC grant GR/R62694/01. Part of this work was written during a visit of JMF to the Erwin Schrödinger Institute to participate in the programme on String theory on curved backgrounds and a subsequent visit to the IHÉS to participate in the Avant Strings workshop. It is a pleasure to thank both institutions for their support and hospitality, and especially Andreas Recknagel and Jean-Pierre Bourguignon for the respective invitations.

PM would like to thank Luis Alvarez-Gaumé, Matthias Blau, Bert Janssen, Martin O'Loughlin and Tomás Ortín for fruitful discussions and Josefina Millán Jiménez for her (library) support. PM would like to thank el Instituto de Física Teórica (Madrid) and het Instituut voor Theoretische Fysica (Leuven) for their support and hospitality.

The research of SP is funded in part by an EPSRC Postgraduate studentship.

\section{Appendix A. Clifford algebra conventions}

Our Clifford algebra conventions mostly follow the book [4], but we will review them here briefly.

Let $\mathbb{R}^{s, t}$ denote the real $(s+t)$-dimensional vector space with inner product obtained from the norm

$$
|\boldsymbol{x}|^{2}=-\left(x^{1}\right)^{2}-\cdots-\left(x^{t}\right)^{2}+\left(x^{t+1}\right)^{2}+\cdots+\left(x^{t+s}\right)^{2},
$$

for $\boldsymbol{x}=\left(x^{1}, \ldots, x^{s+t}\right) \in \mathbb{R}^{s, t}$. By definition the real Clifford algebra $\mathrm{C} \ell(s, t)$ is generated by $\mathbb{R}^{s, t}$ (and the identity $\mathbb{1}$ ) subject to the Clifford relation

$$
\boldsymbol{x} \cdot \boldsymbol{x}=-|\boldsymbol{x}|^{2} \mathbb{1},
$$

where we ask the reader to pay close attention to the sign!

We are interested in eleven-dimensional lorentzian signature: $\mathbb{R}^{1,10}$. As a real associative algebra, $\mathrm{C} \ell(1,10)$ is isomorphic to two copies of the algebra of $32 \times 32$ real matrices. This means that there are (up to isomorphism) two irreducible representations $\mathfrak{M}_{ \pm}$, which are real and thirty-two dimensional. They are distinguished by 
the action of the generator of the centre of $\mathrm{C} \ell(1,10)$, which is realised geometrically by the volume form $\boldsymbol{\nu}$ of $\mathbb{R}^{1,10}$.

The Clifford algebra $\mathrm{C} \ell(1,10)$ is isomorphic as a real vector space (but not as an algebra) to the exterior algebra $\Lambda \mathbb{R}^{1,10}$. In this way, elements of $\Lambda \mathbb{R}^{1,10}$ can act on $\mathfrak{M}_{ \pm}$.

Now let $(M, g)$ be a lorentzian eleven-dimensional manifold, with signature $(1,10)$. We can choose local orthonormal frames for the tangent bundle $T M$ and dual coframes for the cotangent bundle $T^{*} M$. Relative to such a coframe, each cotangent space is isomorphic to $\mathbb{R}^{1,10}$ as an inner product space and we can construct at each point a Clifford algebra $\mathrm{C} \ell(1,10)$. As we let the point vary, these algebras patch up nicely to yield a bundle $\mathrm{C} \ell\left(T^{*} M\right)$ of Clifford algebras which, as a vector bundle, is isomorphic to $\Lambda T^{*} M$. The isomorphism $\Lambda \mathbb{R}^{1,10} \cong \mathrm{C} \ell(1,10)$ also extends to give a bundle isomorphism $\Lambda T^{*} M \cong \mathrm{C} \ell\left(T^{*} M\right)$.

If in addition $(M, g)$ is spin, then there are (not necessarily unique) vector bundles $\mathcal{S}_{ \pm}$associated to each the irreducible representations $\mathfrak{M}_{ \pm}$of $\mathrm{C} \ell(1,10)$. These are bundles of modules over the Clifford bundle $\mathrm{C} \ell\left(T^{*} M\right)$. Differential formsthat is, sections of $\Lambda T^{*} M$-act naturally on sections of $\mathcal{S}_{ \pm}$via the isomorphism $\Lambda T^{*} M \rightarrow \mathrm{C} \ell\left(T^{*} M\right)$ and the natural pointwise action of $\mathrm{C} \ell\left(T^{*} M\right)$ on $\mathcal{S}_{ \pm}$.

In this paper we will have ample opportunity to compute Clifford products of differential forms acting on sections of $\mathcal{S}_{ \pm}$. We collect here some useful formulae.

If $X$ is a vector and $\omega$ a $p$-form, then

$$
X^{b} \cdot \omega=X^{b} \wedge \omega-\iota_{X} \omega
$$

and

$$
\omega \cdot X^{b}=(-1)^{p}\left(X^{b} \wedge \omega+\iota_{X} \omega\right)
$$

where $b$ is the musical isomorphism from vectors to one-forms induced by the metric; that is, the one-form $X^{b}$ is defined by $X^{b}(Y)=\langle X, Y\rangle$ for every vector $Y$. Iterating these identities we find, for example,

$$
\omega \cdot\left(X^{b} \wedge Y^{b}\right)=X^{b} \wedge Y^{b} \wedge \omega+\iota_{X} \iota_{Y} \omega+X^{b} \wedge \iota_{Y} \omega-Y^{b} \wedge \iota_{X} \omega,
$$

and

$$
\left(X^{b} \wedge Y^{b}\right) \cdot \omega=X^{b} \wedge Y^{b} \wedge \omega+\iota_{X} \iota_{Y} \omega-X^{b} \wedge \iota_{Y} \omega+Y^{b} \wedge \iota_{X} \omega .
$$

If $\omega$ is a $p$-form and $\star \omega$ its Hodge dual, then their Clifford actions are related by

$$
\star \omega=(-1)^{p(p+1) / 2} \omega \cdot \boldsymbol{\nu},
$$

where $\boldsymbol{\nu}$ is the volume form.

The bundles $\mathcal{S}_{ \pm}$inherit from $\mathfrak{M}_{ \pm}$a symplectic structure which is compatible with the action of the Clifford algebra; that is, the Clifford endomorphisms corresponding to 1 -forms (equivalently, vectors) are skew-symmetric:

$$
\left(\varepsilon_{1}, v^{b} \cdot \varepsilon_{2}\right)=-\left(v^{b} \cdot \varepsilon_{1}, \varepsilon_{2}\right) .
$$

In turn, this identity implies that the bilinear form

$$
\beta_{v}\left(\varepsilon_{1}, \varepsilon_{2}\right)=\left(\varepsilon_{1}, v^{b} \cdot \varepsilon_{2}\right)
$$

associated to the vector $v$ is symmetric.

More generally, if $\omega$ is a $p$-form, we will let $\omega^{*}$ denote its adjoint with respect to this symplectic structure; that is,

$$
\left(\omega \cdot \varepsilon_{1}, \varepsilon_{2}\right)=\left(\varepsilon_{1}, \omega^{*} \cdot \varepsilon_{2}\right) \text {. }
$$

Explicitly, one finds that

$$
\omega^{*}=(-1)^{p(p+1) / 2} \omega,
$$

whence 1-forms, 2-forms and 5-forms (and their Hodge duals) preserve the symplectic structure. Indeed, $\mathfrak{s p}(32, \mathbb{R})=\Lambda^{1} \oplus \Lambda^{2} \oplus \Lambda^{5}$ under $\mathfrak{s o}(1,10)$. 


\section{Appendix B. Homogeneity of some 16+ Discrete Quotients}

The possible Kaluza-Klein reductions (by one-parameter subgroups) of the maximally supersymmetric Freund-Rubin backgrounds of eleven-dimensional and type IIB supergravities have been classified in 15. Associated to these reductions, there are discrete quotients by a cyclic subgroup. Two of these reductions gave rise to backgrounds with more than 16 supercharges and the same is true for the associated quotients. In this appendix we will show that for every $N>1$ there is a $\mathbb{Z}_{N}$-quotient $\mathrm{AdS}_{4} \times\left(S^{7} / \mathbb{Z}_{N}\right)$ with 24 supercharges, and that there is a a two-parameter family of $\mathbb{Z}$-quotients $\left(\mathrm{AdS}_{4} \times S^{7}\right) / \mathbb{Z}$ with 18 supercharges. We will then demonstrate that these quotients remain homogeneous.

B.1. A family of $\nu=\frac{3}{4}$ quotients. This family of backgrounds has the form $\mathrm{AdS}_{4} \times\left(S^{7} / \Gamma\right)$ where $\Gamma$ is a finite cyclic subgroup of $\mathrm{SO}(8)$. Since $\mathrm{SO}(8)$ is compact, the exponential map is surjective and $\Gamma$ will be generated by an element $\gamma$ in the image of the exponential map. Let us identify the Lie algebra $\mathfrak{s o}(8)$ with the $8 \times 8$ skew-symmetric real matrices. Then consider the element $J \in \mathfrak{s o}(8)$ given by

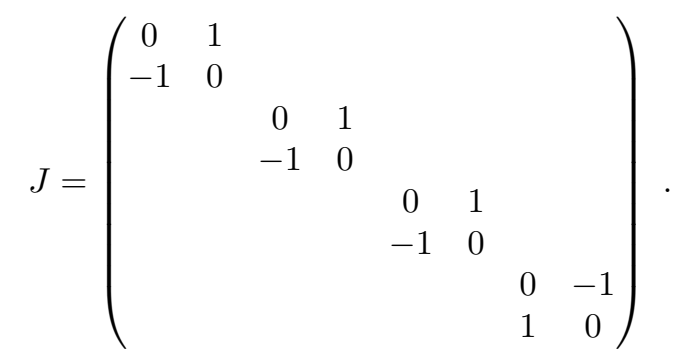

Then consider $\gamma=\exp (2 \pi J / N) \in \mathrm{SO}(8)$. Explicitly,

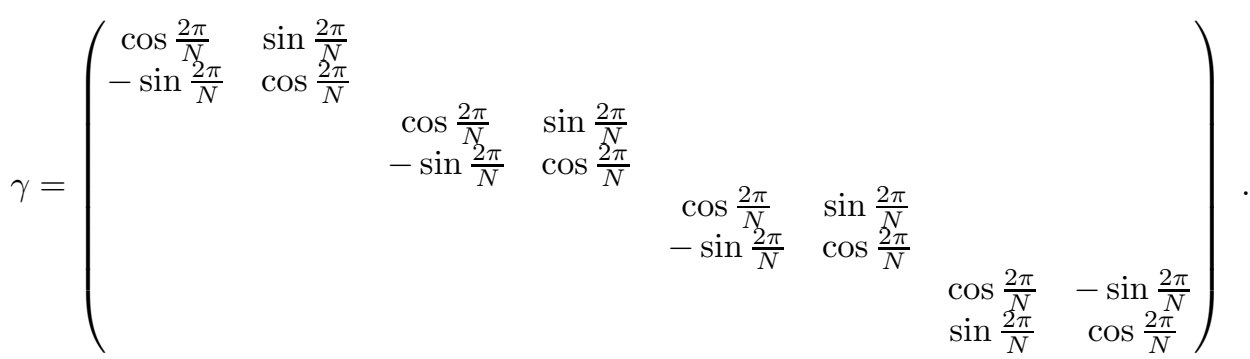

Clearly $\gamma^{N}=1$ and hence it generates a $\mathbb{Z}_{N}$ subgroup of $\operatorname{SO}(8)$. This subgroup acts freely on $S^{7}$ and the resulting quotient is a smooth lens space. The element

$$
\widehat{\gamma}=\exp \left(\frac{\pi}{N} \Gamma_{12}\right) \exp \left(\frac{\pi}{N} \Gamma_{34}\right) \exp \left(\frac{\pi}{N} \Gamma_{56}\right) \exp \left(-\frac{\pi}{N} \Gamma_{78}\right) \in \operatorname{Spin}(8)
$$

is the spin lift of $\gamma$ and clearly obeys $\widehat{\gamma}^{N}=1$, whence as explained in [15, 48, $\Gamma$ lifts to $\operatorname{Spin}(8)$ making the quotient lens space into a spin manifold.

The Killing spinors which survive to the quotient are the $\Gamma$-invariant Killing spinors on $\mathrm{AdS}_{4} \times S^{7}$. As reviewed in [49] for general Freund-Rubin backgrounds, the Killing spinors on $\mathrm{AdS}_{4} \times S^{7}$ are given by tensor products of geometric Killing spinors of $\mathrm{AdS}_{4}$ and $S^{7}$. Since $\Gamma$ only acts on the sphere, we will concentrate on the sphere. The cone construction of [50] relates the geometric Killing spinors on $S^{7}$ to a chiral spinor representation of $\operatorname{Spin}(8)$. In our conventions, this is the spinor representation of negative chirality, which under the action of $\gamma$ is seen to have six zero weights, as explained in more detail in [15, Section 6.2.1]. This implies that the quotient background preserves a fraction $\nu=\frac{6}{8}=\frac{3}{4}$ of the supersymmetry.

We now show that the quotient is homogeneous, but first some general remarks. Let $(M, g)$ have isometry group $G$ and let $\Gamma \subset G$ be a discrete subgroup acting freely on $M$ with a smooth quotient $M / \Gamma$. Not all the isometries of $M$ will descend 
to isometries in the quotient. Indeed, a necessary and sufficient condition for an isometry $g \in G$ to act on $M / \Gamma$ is that if two points $p, q \in M$ are in the same $\Gamma$-orbit, so are their images $g \cdot p, g \cdot q$. The subgroup of $G$ thus defined is the normaliser

$$
N_{\Gamma}=\left\{g \in G \mid g \gamma g^{-1} \in \Gamma \quad \forall \gamma \in \Gamma\right\}
$$

of $\Gamma$ in $G$. Since $\Gamma$ is discrete, the connected component of $N_{\Gamma}$ containing the identity is the centraliser

$$
Z_{\Gamma}=\left\{g \in G \mid g \gamma g^{-1}=\gamma \quad \forall \gamma \in \Gamma\right\}
$$

of $\Gamma$ in $G$. To see this, simply consider a path $g(t)$ from the identity to $g$ in the same connected component of $N_{\Gamma}$ and consider its action on any $\gamma \in \Gamma$. Since $g(t) \in N_{\Gamma}$ for all $t$, we have that $g(t) \gamma g(t)^{-1} \in \Gamma$ for all $t$. Since $\Gamma$ is discrete, continuity means that this has to be the same element of $\Gamma$ for all $t$, but it is $\gamma$ itself when $t=0$.

For the case at hand, the centraliser $Z_{\Gamma}$ is the subgroup of $\mathrm{SO}(8)$ which commutes with the matrix $J$ in (27). Now $J$ is a complex structure and the subgroup thus defined is isomorphic to $\mathrm{U}(4)$, which still acts transitively on $S^{7}$ (with isotropy $\mathrm{U}(3)$ ) and hence will continue to do so in the lens space $S^{7} / \Gamma$. In other words, $S^{7} / \Gamma$ is homogeneous, and hence so is $\operatorname{AdS}_{4} \times\left(S^{7} / \Gamma\right)$.

The Killing superalgebra of the above solution must be a sub-superalgebra of the superalgebra $\mathfrak{o s p}(8 \mid 2, \mathbb{R})$ corresponding to $\operatorname{AdS}_{4} \times S^{7}$. In fact, it is not hard to see that the superalgebra is $\mathfrak{u}(1) \oplus \mathfrak{o s p}(6 \mid 2, \mathbb{R})$, which is a regular maximal subsuperalgebra of $\mathfrak{o s p}(8 \mid 2, \mathbb{R})$ [51. This means that only the $\mathfrak{s u}(4)$ is generated by Killing spinors, but since this acts transitively on $S^{7}$, and will continue to do so on $S^{7} / \Gamma$, we see that also in this case supersymmetry is responsible for homogeneity.

B.2. A family of $\nu=\frac{9}{16}$ quotients. This family of quotients is slightly more involved than the previous one, since the group $\Gamma$ defining the quotient acts on both $\mathrm{AdS}_{4}$ and on $S^{7}$. Let $J \in \mathfrak{s o}(8)$ be as in (27). The isometry algebra of $\mathrm{AdS}_{4}$ is $\mathfrak{s o}(2,3)$, which we can identify with the $5 \times 5$ real matrices which are skew-symmetric relative to a metric $\eta$ of signature $(2,3)$. Let us take $\eta$ to be diagonal with entries $(-1,-1,1,1,1)$ and let $L \in \mathfrak{s o}(2,3)$ be the following matrix

$$
L=\left(\begin{array}{ccccc}
0 & 1 & 1 & 0 & 0 \\
-1 & 0 & 0 & -1 & 0 \\
1 & 0 & 0 & 1 & 0 \\
0 & -1 & -1 & 0 & 0 \\
0 & 0 & 0 & 0 & 0
\end{array}\right)
$$

Now let $\alpha, \beta$ be positive real numbers and consider the element

$$
\gamma=\exp (\alpha L+\beta J) \in \widetilde{\mathrm{SO}}(2,3) \times \mathrm{SO}(8),
$$

where $\widetilde{\mathrm{SO}}(2,3)$, the isometry group of $\mathrm{AdS}_{4}$, is an infinite cyclic cover of $\mathrm{SO}(2,3)$, as discussed in detail in [15, Section 5.1.2]. This element $\gamma$ generates an infinite cyclic subgroup which, as shown in [15, Section 6.2.4], acts freely on $\operatorname{AdS}_{4} \times S^{7}$. The calculations in [15, Section 6.2.4], though written for the continuous $\mathbb{R}$-quotients, show that these $\mathbb{Z}$-quotients preserve a fraction $\nu=\frac{9}{16}$ of the supersymmetry. Indeed, notice that $J$ is as above and we have already seen that it preserves $\frac{3}{4}$ of the $S^{7}$-supersymmetry. Notice that $L^{2}=0$ and that the same analysis as in [15, Section 6.2.4] shows that $L$ preserves another $\frac{3}{4}$ of the $\mathrm{AdS}_{4}$ supersymmetry. Thus we see that there are $6 \Gamma$-invariant Killing spinors on $S^{7}$ and 3 on $\mathrm{AdS}_{4}$ for a total of 18 supercharges in the quotient.

It is however clear that there are, as far as supersymmetry is concerned, two special cases in this family: $\alpha=0$ and $\beta=0$. In both cases the fraction of preserved supersymmetry is $\nu=\frac{3}{4}$, and the geometry corresponds to $\operatorname{AdS}_{4} \times S^{7} / \Gamma$, 
respectively $\mathrm{AdS}_{4} / \Gamma \times S^{7}$. This last case was not treated in [15] since the associated Killing vector has zero norm, whereas [15] focuses on spacelike quotients.

To show homogeneity of the quotient we proceed as before and show that the centraliser $Z_{\Gamma}$ acts transitively already before taking the quotient. The centraliser is the product of the centralisers of the projections of $\Gamma$ to $\widetilde{\mathrm{SO}}(2,3)$ and $\mathrm{SO}(8)$ respectively. We already know from the previous section that the $\mathrm{SO}(8)$-factor is $\mathrm{U}(4)$. The $\widetilde{\mathrm{SO}}(2,3)$-factor of the centraliser is easier to describe infinitesimally; that is, we will describe its Lie algebra which has the form of a semidirect product

$$
\mathfrak{k}=\mathfrak{s l}(2, \mathbb{R})_{+} \ltimes \mathfrak{h}_{3},
$$

where $\mathfrak{s l}(2, \mathbb{R})_{+}$is the self-dual $\mathfrak{s l}(2, \mathbb{R})$ in $\mathfrak{s o}(2,2) \cong \mathfrak{s l}(2, \mathbb{R})_{+} \oplus \mathfrak{s l}(2, \mathbb{R})_{-}$and $\mathfrak{h}_{3}$ is a three-dimensional Heisenberg algebra where the central element is precisely the element $L$ in (28). (Notice that $L$ belongs to $\mathfrak{s l}(2, \mathbb{R})_{-}$.) It is not hard to show that the subgroup $K \subset \mathrm{SO}(2,3)$ with Lie algebra $\mathfrak{k}$ acts transitively on the quadric $-x_{1}^{2}-x_{2}^{2}+x_{3}^{2}+x_{4}^{2}+x_{5}^{2}=-R^{2}$ in $\mathbb{R}^{2,3}$, whence its infinite cyclic cover $\widetilde{K} \subset \widetilde{\mathrm{SO}}(2,3)$, obtained by extending $K$ by the fundamental group of the quadric, also acts transitively on $\mathrm{AdS}_{4}$. In summary, $\widetilde{K} \times \mathrm{U}(4)$ acts transitively on $\mathrm{AdS}_{4} \times S^{7}$ and hence does so on the quotient.

The Killing superalgebra can readily be found by projection, but for definiteness let us discuss the case $\beta=0$. It can then be seen that under $\mathfrak{s l}(2, \mathbb{R}) \oplus \mathfrak{s o}(8) \subset$ $\mathfrak{s o}(2,3) \oplus \mathfrak{s o}(8)$, the invariant Killing spinors transform as $(\underline{\mathbf{2}}, \underline{\mathbf{8}}) \oplus(\underline{\mathbf{1}}, \underline{\mathbf{8}})$. The $\mathfrak{s l}(2, \mathbb{R})$-singlets combine with the $\mathfrak{h}_{3}$ into a Heisenberg superalgebra $\mathfrak{h}_{3 \mid 8}$, meaning that there are 2 bosonic and 8 fermionic creation and annihilation generators, which is a super-ideal of the full superalgebra. The $(\underline{\mathbf{2}}, \underline{\mathbf{8}})$-spinors together with the $\mathfrak{s o}(8)$ and the $\mathfrak{s l}(2, \mathbb{R})$ subalgebras, can be seen to form the algebra osp $(8 \mid 1, \mathbb{R})$, making the full superalgebra $\mathfrak{o s p}(8 \mid 1, \mathbb{R}) \ltimes \mathfrak{h}_{3 \mid 8}$. It is clear that in the general case, the necessary projections on the $\mathrm{AdS}_{4}$ and the $S^{7}$ part are done independently, so that we can combine the above results with the results in Appendix B.1. only to find that in the generic case the Killing superalgebra is $\mathfrak{u}(1) \oplus\left(\mathfrak{o s p}(6 \mid 1, \mathbb{R}) \ltimes \mathfrak{h}_{3 \mid 6}\right)$.

\section{Appendix C. A plane WaVe solution With 22 supercharges}

In 23] it was shown that the Penrose limit of the M-theory Gödel solution generates a one parameter family of wave solutions that interpolates between two Cahen-Wallach (CW) spaces. This family generically preserves 20 supersymmetries which at one CW-point is enhanced to 24. The reasoning of 23] can of course also be applied to the other 16+ Gödel solutions presented in [17], and for completeness we will discuss the resulting non-symmetric plane wave solutions.

Reference [17 finds three 16+ Gödel solutions: the above mentioned M-theory Gödel solution which preserves 20 supersymmetries, an $n=4$ case which also preserves 20 supersymmetries, and finally the $n=5$ case which preserves 18 supersymmetries. The Penrose limit of the $n=5$ is actually a CW-space, and as such ought to be known.

The $n=4$ Gödel solution can be obtained form the type IIB maximally supersymmetric BFHP wave [30] by T-duality and oxidation to eleven dimensions. Its Penrose limit reads

$$
\begin{gathered}
g=2 d u\left(d v-\left[\frac{1}{2} \beta^{2} \vec{x}_{(3 \rightarrow 8)}^{2}+2 \beta^{2} x_{2}^{2}\right] d u-2 \beta p x_{2} d x_{1}\right)+d \vec{x}_{(1 \rightarrow 9)}^{2} \\
F=2 \beta d u \wedge\left(d x^{129}+p d x^{349}+p d x^{569}+p d x^{789}+2 \sqrt{1-p^{2}} d x^{278}\right) .
\end{gathered}
$$

As for the solution in 23] there are two values for which the above solution becomes a Cahen-Wallach space: $p=0$ where the solution preserves 24 supersymmetries, and $p=1$ where one finds 22 supersymmetries. For $p>0$, the necessary 
projection operator onto the extra supersymmetries is given by

$$
\frac{1}{8}\left(1+\sqrt{1-p^{2}} \gamma^{1789}-p \gamma^{9}\right)\left(3+\gamma^{3456}+\gamma^{3478}+\gamma^{5678}\right),
$$

where $\gamma$ are the generators of the transverse $\mathrm{C} \ell(0,9)$ and from which one can see that this solution has 6 extra supersymmetries, for a total of 22 . For $p=0$ the relevant projector is $\frac{1}{2}\left(1+\gamma^{1789}\right)$, which shows that there are now 8 extra supersymmetries.

\section{REFERENCES}

[1] M. Duff and J. Liu, "Hidden spacetime symmetries and generalized holonomy in Mtheory," Nucl. Phys. B674 (2003) 217-230, arXiv:hep-th/0303140

[2] C. M. Hull, "Holonomy and symmetry in M-theory," arXiv:hep-th/0305039

[3] J. M. Figueroa-O'Farrill and G. Papadopoulos, "Maximal supersymmetric solutions of tenand eleven-dimensional supergravity," J. High Energy Phys. 03 (2003) 048, arXiv:hep-th/0211089

[4] W. Nahm, "Supersymmetries and their representations," Nucl. Phys. B135 (1978) 149-166.

[5] E. Cremmer, B. Julia, and J. Scherk, "Supergravity in eleven dimensions," Phys. Lett. 76B (1978) 409-412.

[6] C. M. Hull, "Exact pp-wave solutions of eleven-dimensional supergravity," Phys. Lett. 139B (1984) 39-41.

[7] M. Duff and K. Stelle, "Multi-membrane solutions of D=11 supergravity," Phys. Lett. 253B (1991) 113-118.

[8] R. Güven, "Black $p$-brane solutions of $D=11$ supergravity theory," Phys. Lett. 276B (1992) 49-55.

[9] R. Sorkin, "Kaluza-Klein monopole," Phys. Rev. Lett. 51 (1983) 87-90.

[10] D. Gross and M. Perry, "Magnetic monopoles in Kaluza-Klein theories," Nucl. Phys. B226 (1983) 29.

[11] S. Han and I. Koh, " $N=4$ remaining supersymmetry in a Kaluza-Klein monopole background in D=11 supergravity theory," Phys. Rev. D31 (1985) 2503.

[12] P. Freund and M. Rubin, "Dynamics of dimensional reduction," Phys. Lett. B97 (1980) $233-235$.

[13] J. Kowalski-Glikman, "Vacuum states in supersymmetric Kaluza-Klein theory," Phys. Lett. 134B (1984) 194-196.

[14] J. M. Figueroa-O'Farrill and G. Papadopoulos, "Homogeneous fluxes, branes and a maximally supersymmetric solution of M-theory," J. High Energy Phys. 06 (2001) 036, arXiv: hep-th/0105308

[15] J. M. Figueroa-O'Farrill and J. Simón, "Supersymmetric Kaluza-Klein reductions of AdS backgrounds," Adv. Theor. Math. Phys. 8 (2004) 217-317, arXiv:hep-th/0401206

[16] J. P. Gauntlett, J. Gutowski, C. M. Hull, S. Pakis, and H. Reall, "All supersymmetric solutions of minimal supergravity in five dimensions," Class. Quant. Grav. 20 (2003) 4587-4634, arXiv:hep-th/0209114

[17] T. Harmark and T. Takayanagi, "Supersymmetric Gödel universes in string theory," Nucl. Phys. B662 (2003) 3-39, arXiv:hep-th/0301206

[18] M. Cvetič, H. Lü, and C. Pope, "Penrose limits, pp-waves and deformed M2-branes," Phys. Rev. D69 (2004) 046003, arXiv: hep-th/0203082

[19] J. Michelson, "(Twisted) toroidal compactification of pp-waves," Phys. Rev. D66 (2002) 066002, arXiv:hep-th/0203140

[20] J. P. Gauntlett and C. M. Hull, "pp-waves in 11-dimensions with extra supersymmetry," J. High Energy Phys. 06 (2002) 013, arXiv:hep-th/0203255.

[21] M. Cvetič, H. Lü, and C. Pope, "M-theory pp-waves, Penrose limits and supernumerary supersymmetries," Nucl. Phys. B644 (2002) 65-84, arXiv:hep-th/0203229

[22] J. Michelson, "A pp-wave with 26 supercharges," Class. Quant. Grav. 19 (2002), no. 23, 5935-5949, arXiv:hep-th/0206204

[23] M. Blau, P. Meessen, and M. O’Loughlin, "Gödel, Penrose, anti-Mach: extra supersymmetries of time-dependent plane waves," J. High Energy Phys. 09 (2003) 072, arXiv:hep-th/0306161

[24] C. Patricot, "Kaigorodov spaces and their Penrose limits," Class. Quant. Grav. 20 (2003) 2087-2102, arXiv:hep-th/0302073

[25] R. Geroch, "Limits of spacetimes," Comm. Math. Phys. 13 (1969) 180-193.

[26] R. Penrose, "Any space-time has a plane wave as a limit," in Differential geometry and relativity, pp. 271-275. Reidel, Dordrecht, 1976. 
[27] R. Güven, "Plane wave limits and T-duality," Phys. Lett. B482 (2000) 255-263, arXiv:hep-th/0005061

[28] M. Blau, J. M. Figueroa-O'Farrill, and G. Papadopoulos, "Penrose limits, supergravity and brane dynamics," Class. Quant. Grav. 19 (2002) 4753-4805, arXiv:hep-th/0202111

[29] M. Blau, J. M. Figueroa-O'Farrill, C. M. Hull, and G. Papadopoulos, "Penrose limits and maximal supersymmetry," Class. Quant. Grav. 19 (2002) L87-L95, arXiv:hep-th/0201081

[30] M. Blau, J. M. Figueroa-O'Farrill, C. M. Hull, and G. Papadopoulos, "A new maximally supersymmetric background of type IIB superstring theory," J. High Energy Phys. 01 (2002) 047, arXiv:hep-th/0110242

[31] P. Meessen, "A small eprint on pp-wave vacua in 6 and 5 dimensions," Phys. Rev. D65 (2002) 087501, arXiv:hep-th/0111031.

[32] B. S. Acharya, J. M. Figueroa-O'Farrill, C. M. Hull, and B. Spence, "Branes at conical singularities and holography," Adv. Theor. Math. Phys. 2 (1998) 1249-1286, arXiv:hep-th/9808014

[33] J. P. Gauntlett, R. Myers, and P. K. Townsend, "Supersymmetry of rotating branes," Phys. Rev. D59 (1999) 025001, arXiv:hep-th/9809065

[34] J. P. Gauntlett, R. Myers, and P. K. Townsend, "Black holes of $D=5$ supergravity," Class. Quant. Grav. 16 (1999) 1-21, arXiv:hep-th/9810204

[35] P. K. Townsend, "Killing spinors, supersymmetries and rotating intersecting branes," in Novelties in string theory (Göteborg, 1998), pp. 177-182. World Sci. Publishing, River Edge, NJ, 1999. arXiv:hep-th/9901102

[36] J. M. Figueroa-O'Farrill, "On the supersymmetries of Anti-de Sitter vacua," Class. Quant. Grav. 16 (1999) 2043-2055, arXiv:hep-th/9902066

[37] N. Alonso-Alberca, E. Lozano-Tellechea, and T. Ortín, "Geometric construction of Killing spinors and supersymmetry algebras in homogeneous spacetimes," Class. Quant. Grav. 19 (2002) 6009-6024, arXiv:hep-th/0208158

[38] J. M. Figueroa-O'Farrill, "Maximal supersymmetry in ten and eleven dimensions," in Special geometric structures in String Theory, D. V. Alekseevsky, V. Cortés, C. Devchand, and A. V. Proeyen, eds. EMIS, Bonn, September, 2001. arXiv:math.DG/0109162

[39] B. Kostant, "Holonomy and the Lie algebra of infinitesimal motions of a riemannian manifold," Trans. Am. Math. Soc. 80 (1955) 528-542.

[40] Y. Kosmann, "Dérivées de Lie des spineurs," Annali di Mat. Pura Appl. (IV) 91 (1972) 317-395.

[41] J. P. Gauntlett and S. Pakis, "The geometry of $D=11$ Killing spinors," J. High Energy Phys. 04 (2003) 039, arXiv:hep-th/0212008

[42] J. M. Figueroa-O'Farrill, "Breaking the M-waves," Class. Quant. Grav. 17 (2000) 2925-2947, arXiv:hep-th/9904124

[43] M. Wang, "Parallel spinors and parallel forms," Ann. Global Anal. Geom. 7 (1989), no. 1, $59-68$.

[44] D. Brecher and M. Perry, "Ricci-flat branes," Nucl. Phys. B566 (2000) 151-172, arXiv:hep-th/9908018

[45] J. M. Figueroa-O'Farrill, "More Ricci-flat branes," Phys. Lett. 471B (2000) 128-132, arXiv:hep-th/9910086

[46] A. Batrachenko, M. J. Duff, J. T. Liu, and W. Y. Wen, "Generalized holonomy of M-theory vacua," arXiv:hep-th/0312165

[47] H. B. Lawson and M.-L. Michelsohn, Spin geometry. Princeton University Press, 1989.

[48] J. M. Figueroa-O'Farrill, O. Madden, S. Ross, and J. Simón, "Quotients of $\operatorname{AdS}_{p+1} \times S^{q}$ : causally well-behaved spaces and black holes," Phys. Rev. D69 (2004) 124026, arXiv:hep-th/0402094

[49] J. M. Figueroa-O'Farrill, F. Leitner, and J. Simón, "Supersymmetric Freund-Rubin backgrounds." Unfinished draft, 2003.

[50] C. Bär, "Real Killing spinors and holonomy," Comm. Math. Phys. 154 (1993) 509-521.

[51] L. Frappat, A. Sciarrino, and P. Sorba, Dictionary on Lie algebras and superalgebras. Academic Press Inc., San Diego, CA, 2000. hep-th/9607161

(JMF,SP) School of Mathematics, University of Edinburgh

E-mail address: J.M.Figueroa@ed.ac.uk, S.A.R.Philip@sms.ed.ac.uk

(PM) Theory Group, CERN, Geneva, Switzerland

E-mail address: Patrick.Meessen@cern.ch 